\title{
A Retrospective Study on Contact Allergy to Fragrance Mix II and Hydroxyisohexyl 3-Cyclohexene Carboxaldehyde By the International Contact Dermatitis Research Group
}

Magnus Bruze, MD, PhD, * Iris Ale, MD, DM, † Klaus E. Andersen, DMSc, $\$$ Alicia Cannavó, MD, $\S$

Thomas Diepgen, MD, PhD, || Peter Elsner, MD, PhD, $\uparrow$ Chee-Leok Goh, MD, MRCP, MMed,\#

Margarida Gonçalo, MD, PhD,** An Goossens, PhD, †† John McFadden, MD, DM, $+\uparrow+$ Rosemary Nixon, FACD, $\S \S$

Pailin Puangpet, MD, ||| Denis Sasseville, MD, FRCPC, $\$$ |

and On behalf of the International Contact Dermatitis Research Group

Background: Fragrance mix II (FM II) is included in the baseline patch test series recommended by the International Contact Dermatitis Research Group (ICDRG). Hydroxyisohexyl 3-cyclohexene carboxaldehyde (HICC) is the most important sensitizer of the 6 fragrance materials included in FM II. Besides being a part of FM II, HICC is also tested separately in the ICDRG baseline series.

Objectives: The aim of the study was to investigate the prevalence of contact allergy to FM II and HICC in 2012-2016 with a focus on simultaneous reactions and the percentage of missed contact allergy to HICC provided that only FM II had been tested.

Patients and Methods: A total of 25,019 consecutive dermatitis patients in 13 dermatology clinics representing 12 countries in 5 continents were patch tested with FM II and HICC in the baseline series.

Results: Contact allergy to FM II and HICC was found in $3.9 \%$ and $1.6 \%$, respectively. For FM II, the frequency varied from $1.5 \%$ to $7.6 \%$ in different centers. The corresponding range for HICC was $0.2 \%$ to $3.6 \%$. Simultaneous contact allergy to FM II and $\mathrm{HICC}$ was noted in $1.4 \%$ with the range $0.2 \%$ to $2.6 \%$. Seventy-seven patients $(0.31 \%)$ with contact allergy to HICC did not test positively to FM II. The range for missed HICC allergy by testing only FM II in the different centers would be $0.04 \%$ to

From the ${ }^{*}$ Department of Occupational and Environmental Dermatology, Lund University, Skåne University Hospital, Malmö, Sweden; †Allergy Center and Department of Dermatology, University Hospital, Republic University of Uruguay, Montevideo; $\ddagger$ Department of Dermatology and Allergy Centre, Odense University Hospital, University of Southern Denmark, Denmark; §Dermatology Department Buenos Aires University, Clinicas Hospital, Argentina; /|Occupational Dermatology, Ruprecht-Karls-University, Heidelberg, Germany; 9 Department of Dermatology, University Hospital Jena, Germany; \#Department of Dermatology, National Skin Center, Singapore; ${ }^{*}$ Department of Dermatology, University Hospital and Faculty of Medicine, University of Coimbra, Portugal; $\dagger \dagger$ Contact Allergy Unit, Department of Dermatology, University Hospital K.U. Leuven, Belgium; $¥ ¥$ St John’s Institute of Dermatology, Contact Dermatitis Clinic, St Thomas' Hospital, London, United Kingdom; \$§Occupational Dermatology Research and Education Centre, Skin and Cancer Foundation Inc, Melbourne, Australia; |||Institute of Dermatology, Bangkok, Thailand; and 99 Division of Dermatology, McGill University Health Centre, Montreal General Hospital, Quebec, Canada.

Address reprint requests to Magnus Bruze, MD, PhD, Department of Occupational Dermatology, Skåne University Hospital, Jan Waldenströms gata 16, S-205 02, Malmo, Sweden.

M.B. is a member of the Expert Panel for Fragrance Safety (http:// fragrancesafetypanel.org/).

The other authors have no funding or conflicts of interest to declare.

DOI: 10.1097/DER.0000000000000545

(C) 2020 American Contact Dermatitis Society. All Rights Reserved.
$0.74 \%$. The ratio between the contact allergy rates for FM II and HICC was similar for all centers, except for Montreal having significantly more contact allergy to FM II than to HICC.

Conclusions: The frequency of missed contact allergy to HICC when testing only with FM II was less than $0.5 \%$, therefore questioning the need to test HICC separately in the ICDRG baseline series.

7 he fragrance substance hydroxyisohexyl 3-cyclohexene 1 carboxaldehyde (HICC) has been a significant contact sensitizer In Europe ${ }^{1-15}$ but not in North America. ${ }^{16,17}$ It has been included in the European baseline series both as a single allergen and as 1 of 6 constituents in fragrance mix II (FM II). ${ }^{18}$ Hydroxyisohexyl 3-cyclohexene carboxaldehyde and FM II are also tested simultaneously in other baseline patch test series including the baseline series of the International Contact Dermatitis Research Group (ICDRG). ${ }^{19}$ A baseline patch test series should regularly be evaluated for possible changes regarding insertion of new sensitizers, removal of old ones, and other changes of preparations. ${ }^{20}$ The ICDRG baseline series is under regular evaluation for possible changes, and contact allergy 


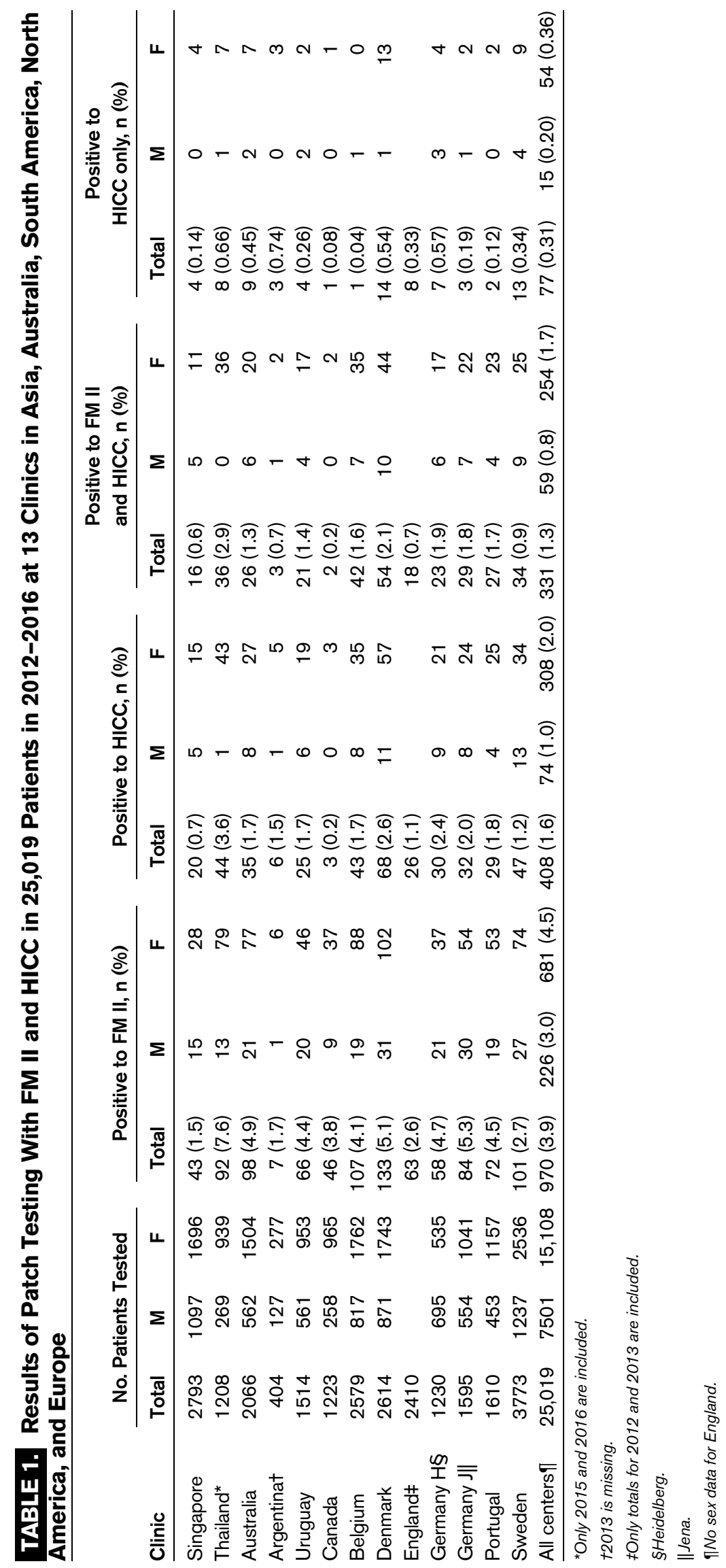


rates are collected from ICDRG departments including simultaneous contact allergies to HICC and FM II. In this study, contact allergy rates to HICC and FM II are presented from 13 clinics in Asia, Australia, South America, North America, and Europe with a focus on missed contact allergy to HICC if only patch testing with FM II was performed.

\section{MATERIALS AND METHODS}

\section{Patients}

Annual contact allergy rates for HICC and FM II have been collected in 25,019 dermatitis patients for 2012 to 2016 from the departments of dermatology/occupational dermatology in Singapore, Bangkok, Melbourne, Buenos Aires, Montevideo, Montreal, Leuven, Odense, London, Heidelberg, Jena, Coimbra, and Malmö (Table 1). Two test preparations with HICC and FM II were included in the respective baseline patch test series.

\section{Patch Testing}

Each clinic has used its routine methodology. Chemotechnique Diagnostics (Vellinge, Sweden) supplied petrolatum preparations of HICC and FM II at 5\% and 16\%, respectively. To avoid evaporation of the fragrance materials, the preparations have been applied to the test chambers/units immediately before application to the patient's back. $^{21,22}$ The occlusion time was 48 hours. Various reading times have been used, but for this study, the results on day (D) 3 or 4 have been considered. The morphology of the test reactions have been classified according to the ICDRG. ${ }^{23}$ Doubtful reactions were not included.

\section{Statistical Calculations}

Sex differences in contact allergy rates were investigated with the $\chi^{2}$ method, 2-sided. Fisher exact test, 2 -sided, was used to investigate differences in distribution of positive reactions to FM II and HICC. The comparison was performed individually for each clinic versus all the others; that is, the distribution of positive reactions to FM II and HICC for 1 clinic was compared with the distribution of these reactions in all other clinics together.

\section{RESULTS}

The contact allergy rates for HICC and FM II as well as the simultaneous positive reactions to HICC and FM II are given in Table 1. Based on all clinics, the contact allergy rates for HICC and FM II were $1.6 \%$ and $3.9 \%$, respectively. The rates for FM II varied between $1.5 \%$ for Singapore and $7.6 \%$ for Bangkok. The range for HICC included the lowest value, $0.2 \%$, for Montreal and the highest one, $3.6 \%$, for Bangkok. Contact allergy was more common in females based on all centers. The double frequency was noted for HICC, $2.0 \%$ versus $1.0 \%$, and $50 \%$ more for FM II, $4.5 \%$ versus $3.0 \%$. Both differences were statistically significant $(P<0.001)$.
For all centers, there were 2.4 times more positive reactions to FM II than to HICC with a range from 1.1 for Buenos Aires and 19.0 for Montreal. There was no statistically different distribution of positive reactions to FM II and HICC in each center except for Montreal $(P<0.001)$.

A total of 408 positive reactions to HICC were registered, and a simultaneous positive reaction to FM II was seen in 331 cases. Thus, 77 positive reaction to HICC $(77 / 408=18.9 \%)$ would have been missed, unless HICC had been tested simultaneously. Missed HICC allergy was thus seen in $0.31 \%$ of all tested patients. The range varied from $0.04 \%$ for Leuven up to the highest value $0.74 \%$ for Buenos Aires, followed by Bangkok with $0.66 \%$.

\section{DISCUSSION}

The contact allergy rates for FM II and HICC are more than 1\% in all centers, except for HICC with $0.7 \%$ and $0.2 \%$ for Singapore and Montreal, respectively. As reported before, there is a female predominance, particularly concerning HICC. The contact allergy rates were similar in all geographical areas, except for Canada (Montreal) with 19 times more contact allergy to FM II as compared with HICC, suggesting a difference in exposure. This has been pointed out earlier as a likely explanation for the low contact allergy rates to HICC in North America. ${ }^{16,17}$

Recently, 16 factors of possible significance for the patch test result were discussed and scored in a publication on how to improve the quality of multicenter patch test studies. ${ }^{24,25}$ Obviously, retrospective multicenter patch test studies, such as the present one, can be expected to have a lower quality as compared with prospective studies. Nevertheless, the present study carries an acceptable quality based on the scoring of the 16 factors. ${ }^{25}$ Among the factors with lower scores, differences in patch test systems and manufacturers of the test preparations as well as lack of patch test reading on day 7, 1 week after the test application, are found. Have these differences in performance any impact on the possibility to draw wellfounded conclusions on the contact allergy rates to FM II and HICC as well as the presence of simultaneous positive patch test reactions to FM II and HICC? To some extent, it is likely between differences in MOAHLFA indices ${ }^{26}$ and exposure conditions that the variation in contact allergy rates can be explained rather than by differences in performing the tests. However, the major aim of this study was to get information about the percentage of missed contact allergy to HICC when tested simultaneously with FM II. Any variation in patch test methodology between centers is expected to affect test results with both patch test allergens in a similar way. The average missed contact allergy for HICC if only testing with FM II was $0.31 \%$ and with all clinics clearly less than $1 \%$ (Table 1). One possible explanation for the negative reactions to FM II in HICC-hypersensitive patients is the lower concentration of HICC in FM II, 2.5\%, as compared with $5 \%$ when tested individually in the baseline series. However, patch testing with a FM II-containing HICC at $5 \%$ within the Swedish Contact Dermatitis Research Group did not improve the diagnostics. ${ }^{12}$ 
Contact allergy rates exceeding $1 \%$ were registered for all 13 clinics for FM II and for all but 2 for HICC (Table 1). Although not systematically compiled in the present study, many of the allergic reactions to FM II and/or HICC were clinically relevant. It therefore seems obvious that both sensitizers should be present in the ICDRG baseline series. ${ }^{20}$ However, the gain by testing a separate sensitizer twice should also be considered. Recently, the ICDRG decided to remove HICC from the ICDRG baseline patch test series because of a too low gain. ${ }^{27}$ Whenever there is a positive reaction to FM II, its ingredients should always be tested separately, provided that the fragrance substances in FM II are labeled on cosmetics in the environment of the tested individual.

\section{REFERENCES}

1. Frosch PJ, Johansen JD, Menné T, et al. Lyral is an important sensitizer in patients sensitive to fragrances. Br J Dermatol 1999;141:1076-1083.

2. Frosch PJ, Pirker C, Rastogi SC, et al. Patch testing with a new fragrance mix detects additional patients sensitive to perfumes and missed by the current fragrance mix. Contact Dermatitis 2005;52:207-215.

3. Frosch PJ, Rastogi SC, Pirker C, et al. Patch testing with a new fragrance mix - reactivity to the individual constituents and chemical detection in relevant cosmetic products. Contact Dermatitis 2005;52:216-225.

4. Nardelli A, Carbonez A, Ottoy W, et al. Frequency of and trends in fragrance allergy over a 15-year period. Contact Dermatitis 2008;58:134-141.

5. Cuesta L, Silvestre JF, Toledo F, et al. Fragrance contact allergy: a 4-year retrospective study. Contact Dermatitis 2010;63:77-84.

6. Krautheim A, Uter W, Frosch P, et al. Patch testing with fragrance mix II: results of the IVDK 2005-2008. Contact Dermatitis 2010;63:262-269.

7. Heisterberg MV, Andersen KE, Avnstorp C, et al. Fragrance mix II in the baseline series contributes significantly to detection of fragrance allergy. Contact Dermatitis 2010;63:270-276.

8. Carvalho R, Maio P, Amaro C, et al. Hydroxyisohexyl 3-cyclohexene carboxaldehyde $\left(\right.$ Lyral $\left.{ }^{\circledR}\right)$ as allergen: experience from a contact dermatitis unit. Cutan Ocul Toxicol 2011;30:249-250.

9. Nardelli A, Carbonez A, Drieghe J, et al. Results of patch testing with fragrance mix 1, fragrance mix 2, and their ingredients, and Myroxylon pereirae and colophonium, over a 21-year period. Contact Dermatitis 2013;68:307-313.

10. Isaksson $M$, Inerot $A$, Lidén $C$, et al. Multicentre patch testing with fragrance mix II and hydroxyisohexyl 3-cyclohexene carboxaldehyde by the Swedish Contact Dermatitis Research Group. Contact Dermatitis 2014;70:187-189.

11. Frosch PJ, Duus Johansen J, Schuttelaar ML, et al, ESSCA Network. Patch test results with fragrance markers of the baseline series-analysis of the European
Surveillance System on Contact Allergies (ESSCA) Network 2009-2012. Contact Dermatitis 2015;73:163-171.

12. Engfeldt M, Hagvall L, Isaksson M, et al. Patch testing with hydroxyisohexyl 3-cyclohexene carboxaldehyde (HICC) - a multicentre study of the Swedish Contact Dermatitis Research Group. Contact Dermatitis 2017;76:34-39.

13. Bennike NH, Zachariae C, Johansen JD. Non-mix fragrances are top sensitizers in consecutive dermatitis patients-a cross-sectional study of the 26 EU-labelled fragrance allergens. Contact Dermatitis 2017;77:270-279.

14. Mowitz M, Svedman C, Zimerson E, et al. Simultaneous patch testing with fragrance mix I, fragrance mix II and their ingredients in southern Sweden between 2009 and 2015. Contact Dermatitis 2017;77:280-287.

15. Ung CY, White JML, White IR, et al. Patch testing with the European baseline series fragrance markers: a 2016 update. Br J Dermatol 2018;178:776-780.

16. Warshaw EM, Belsito DV, Taylor JS, et al. North American Contact Dermatitis Group patch test results: 2009 to 2010. Dermatitis 2013;24:50-59.

17. DeKoven JG, Warshaw EM, Belsito DV, et al. North American Contact Dermatitis Group patch test results 2013-2014. Dermatitis 2017;28:33-46.

18. Bruze M, Andersen KE, Goossens A, ESCD; EECDRG. Recommendation to include fragrance mix 2 and hydroxyisohexyl 3-cyclohexene carboxaldehyde (Lyral) in the European baseline patch test series. Contact Dermatitis 2008; 58:129-133.

19. Alikhan A, Cheng LS, Ale I, et al. Revised minimal baseline series of the International Contact Dermatitis Research Group: evidence-based approach. Dermatitis 2011;22:121-122.

20. Bruze M, Condé-Salazar L, Goossens A, et al. Thoughts on sensitizers in a standard patch test series. The European Society of Contact Dermatitis. Contact Dermatitis 1999;41:241-250.

21. Mowitz M, Zimerson E, Svedman C, et al. Stability of fragrance patch test preparations applied in test chambers. Br J Dermatol 2012;167:822-827.

22. Mowitz M, Svedman C, Zimerson E, et al. Fragrance patch tests prepared in advance may give false-negative reactions. Contact Dermatitis 2014;71:289-294.

23. Fregert S. Manual of Contact Dermatitis. 2nd ed. Copenhagen, Denmark: Munksgaard; 1981.

24. Andersen KE. Multicentre patch test studies: are they worth the effort? Contact Dermatitis 1998;38:222-223.

25. Bruze M. Thoughts on how to improve the quality of multicentre patch test studies. Contact Dermatitis 2016;74:168-174.

26. Uter W, Schwitulla J, Thyssen JP, et al. The 'overall yield' with the baseline series-a useful addition to the array of MOAHLFA factors describing departmental characteristics of patch tested patients. Contact Dermatitis 2011;65:322-328.

27. Isaksson M, Ale I, Andersen KE, et al. Revised baseline series of the International Contact Dermatitis Research Group. Dermatitis 2020. doi: 10.1097/ DER.0000000000000532. [Epub ahead of print]. 